\begin{tabular}{|c|l|}
\hline Title & Remote excitation-tip-enhanced Raman scattering microscopy using silver nanowire \\
\hline Author(s) & Fujita, Y asuhiko; W alke, Peter; De Feyter, Steven; Uji-i, Hiroshi \\
\hline Citation & $\begin{array}{l}\text { Japanese Journal of A pplied Physics (JJA P), 55(8S1), 08NB03 } \\
\text { https://doi.org/L0.7567/JJA.55.08NB03 }\end{array}$ \\
\hline Issue Date & 2016-08 \\
\hline Doc URL & http://hdl.handle.net/2115/66893 \\
\hline Rights & ○ 2016 The Japan Society of A pplied Physics \\
\hline Type & article (author version) \\
\hline File Information & SP15026.pdf \\
\hline
\end{tabular}

Instructions for use 


\section{Remote excitation-tip-enhanced Raman scattering microscopy using silver nanowire}

Yasuhiko Fujita ${ }^{1}$, Peter Walke ${ }^{1}$, Steven De Feyter ${ }^{1}$, and Hiroshi Uji-i ${ }^{1,2}$

${ }^{1}$ KU Leuven, Departement Chemie, Celestijnenlaan 200G-F, 3001 Heverlee, Belgium

${ }^{2}$ RIES, Hokkaido University, Sapporo 001-0020, Japan

E-mail: yasuhiko.fujita@chem.kuleuven.be

Tip-enhanced Raman scattering (TERS) microscopy is a promising technique for use in surface analysis, allowing both topographic and spectroscopic information to be obtained simultaneously at a scale below $10 \mathrm{~nm}$. One proposed method to further improve spatial resolution is the use of propagating surface plasmons as an excitation light source (i.e. remote excitation). However, this requires a specialized tip that can only be fabricated via expensive procedures, such as electron-beam lithography. Here, we propose a new fabrication method for silver nanowire-based tips that are suitable for remote excitation-TERS, removing the need for such techniques. A silver nanowire was fixed onto a Tungsten-tip using a micromanipulator, before gold nanoparticles were attached in a site-specific manner using AC-dielectrophoresis. All the processes were completed using an optical microscope in the ambient. The background intensities in TERS spectra were suppressed with remote excitation relative to the conventional excitation configuration, indicating an increase in TERS sensitivity. 


\section{Introduction}

The ability to fully characterize adsorbed molecules on surfaces is of fundamental importance in a wide variety of technologically relevant research fields, in order to understand how processes at the nanoscale -such as reactions and nano-patterning-come to dictate properties at the macroscale. Knowledge accumulated from this could then translate directly into applications, ranging from advances in battery technology ${ }^{1-3)}$ and solar cells to novel forms of catalysis ${ }^{4-6)}$. The ultimate goal would be for topographic and spectroscopic data to be acquired from the same point with sub-nanometer resolution, and thus the individual contributions of molecules to be established.

Scanning probe microscopy (SPM), such as scanning tunnelling microscopy (STM) or atomic force microscopy (AFM), has been used for more than 30 years to describe the morphology of surfaces. STM in particular possesses a resolution of around 0.1 and $0.01 \mathrm{~nm}$ in the respective lateral and vertical directions, and thus allows routine imaging of molecules on surfaces at the atomic scale ${ }^{7)}$. But the breadth of information such a technique can provide is limited. Different molecular species are usually differentiated by the contrast obtained in images, for which definitive signatures are limited. STM-based spectroscopy, such as scanning tunnelling spectroscopy (STS) or inelastic tunnelling spectroscopy, are powerful tools that do provide additional information, but the interpretation of such data is not always trivial. Further, it is typical for a low-temperature and ultra-high vacuum environment to be required and so their degree of applicability to 'real-world' conditions is limited.

Conversely, laser-based spectroscopies, such as Raman/IR or fluorescence spectroscopy, are widely used to investigate molecular composition, orientation and electronic or vibrational structure. Indeed, the Raman/IR spectrum of a sample strongly depends on the constituents present, meaning that the individual molecular species can be detected. However, the spatial resolution of optical techniques is limited by the diffraction limit of light, approximated to be $0.61 \lambda / N A$, where $\lambda$ and $N A$ are wavelength of incident light and numerical aperture of an objective, respectively. This means that under normal conditions the maximum obtainable resolution using visible excitation light is around $200 \mathrm{~nm}$, which is far from the resolution needed to investigate at the molecular scale.

Tip-enhanced Raman scattering (TERS) microscopy is a new technique developed to couple the high information content afforded by optical techniques, with the molecular level topographic information provided by $\mathrm{SPM}^{8-12)}$. Here, a noble metal SPM tip is irradiated by focused laser light, leading to the excitation of localized surface plasmons resonances (LSPRs) and the introduction of the lightning rod effect, as schematically shown in Fig. 1(a) 
(we name this as 'direct excitation'). A high resolution of around $10 \mathrm{~nm}$ is made possible by the strong electromagnetic-field -which serves to strongly enhance the Raman scattering probability- generated in the vicinity of the tip ${ }^{13,14)}$.

One limitation with TERS is a lack of experimental reproducibility, especially with regards to tip production. The degree of TERS activity is ultimately determined by the structure at the end of the tip, and thus differs from tip to tip -even in the absence of any variability in experimental protocol. As a means to overcome this, we have recently fabricated a new type of TERS tip using chemically-synthesized silver nanowires ${ }^{15)}$. We have previously reported that, thanks to the high-reproducibility of the end-structure of the silver nanowires due to their high crystallinity, almost $100 \%$ of fabricated tips show TERS activity. This is contrasted with results using conventional procedures (such as chemical etching or vacuum deposition), for which a very limited number of tips can provide appreciable TERS enhancement.

Several methods have also been proposed to further improve sensitivity, i.e. resolution, for example by using non-linear excitation ${ }^{16)}$ or by tuning the plasmon resonance frequency by the tunnelling current in STM mode ${ }^{17,18)}$. A further promising way to increase the sensitivity is exciting TERS via propagating surface plasmons, i.e., remote excitation. This can be realized if the excitation light is focused onto an appropriate coupling point (e.g. a periodic grating structure or nanoparticle/nanowire junction) away from the detection spot (tip apex), exciting propagating surface plasmons along the metal structure. We have recently observed that the background intensity in surface-enhanced Raman scattering (SERS) is suppressed with remote excitation relative to direct excitation ${ }^{19-21)}$. This is most likely due to the smaller contribution to the overall spectra of any far-field Raman scattering or photoluminescence emanating from around the focal spot. Furthermore, Berweger et al. have demonstrated that the use of propagating surface plasmons in TERS allows not only for an increase in the sensitivity due to background suppression, but also means that TERS can be conducted in the near-IR region ${ }^{22,23)}$. This has been realized by focusing visible/near-IR laser to a periodic grating structure formed on an electrochemically-etched gold tip by ion-beam milling. Although this is a reliable method to conduct high sensitivity remote excitation-TERS, the requirement of high cost equipment could limit its applicability. Furthermore, given their aforementioned reproducibility, the realization of remote excitation-TERS using chemically-synthesized nobel metal nanowires is of vital importance.

In this contribution, we report a new method for fabricating silver nanowire-based tips 
suitable for remote excitation-TERS (a schematic image is shown in Fig. 1(b)). A silver nanowire on a glass surface was fixed onto a sharpened tungsten tip (W-tip) using a micromanipulator, allowing for the length of the exposed nanowire to be precisely controlled. Gold nanoparticle (s), working as a light coupling point, were attached onto the body of the nanowire in a site-specific manner via alternating-current dielectrophoresis (AC-DEP). It is noteworthy that all processes could be completed using an optical microscope, and so cost-consuming vacuum techniques, such as focused ion beam milling, are not required. Finally, the remote-activity of the fabricated tips was investigated. A tip was approached to a benzenethiol-modified $\mathrm{Au}(111)$ surface under shear-force AFM feedback, allowing TERS spectra taken using both direct and remote excitation to be compared. It was observed that the background intensity with remote excitation was much lower than with direct excitation, indicating the remote-activity of the tips.

\section{Experimental methods}

\subsection{Synthesis of silver nanowire}

The silver nanowires were synthesized using the polyol method as previously reported ${ }^{24)}$. Briefly, after heating up $5 \mathrm{~mL}$ of $0.15 \mathrm{M}$ poly(vinyl pyrrolidone) (PVP) solution in ethylene glycol (EG) at $150{ }^{\circ} \mathrm{C}$ for $15 \mathrm{~min}, 40 \mu \mathrm{L}$ of $\mathrm{CuCl}_{2}(4 \mathrm{mM})$ solution in EG was injected, followed by adding $2.5 \mathrm{~mL}$ of $\mathrm{AgNO}_{3}$ into EG solution drop-wisely under magnetic stirring (600 rpm). Then the solution was kept at $150{ }^{\circ} \mathrm{C}$ for another $1.5 \mathrm{~h}$, obtaining high-yield silver nanowires. These silver nanowires were then washed with ethanol three times. Average diameter and length of silver nanowires are $90 \pm 10 \mathrm{~nm}$ and $10 \pm 2 \mu \mathrm{m}$, respectively.

\section{2 preparation of sample}

First, in order to remove any contamination from the surface, the $\mathrm{Au}(111)$ substrate (purchased from Georg Albert PVD-Beschichtungen) was flame annealed for $60 \mathrm{~s}$ with $1 \mathrm{~Hz}$ and was then cooled under $\mathrm{N}_{2}$ atmosphere. The cleaned substrate was then immediately soaked into $1 \mathrm{mM}$ benzenethiol/ethanol solution for about $12 \mathrm{~h}$, before being washed with MilliQ and ethanol, and dried using $\mathrm{N}_{2}$.

\subsection{TERS setup}

TERS measurements were performed with OmegaScope ${ }^{\text {TM }} 1000$ (AIST-NT). Fig. 2 shows a schematic of our setup. Laser light from a He-Ne laser $(632.8 \mathrm{~nm})$ was reflected by a dichroic mirror (Chroma Z633RDC) and then focused onto the sample surface from the side 
(with an angle of $28^{\circ}$ with respect to the sample surface) by an objective (BD Plan Apo 100x, NA 0.7). The Optical density at the sample surface was about $50 \mathrm{~kW} / \mathrm{cm}^{2}$. Raman scattering was collected with the same objective and directed to a Raman spectrograph (Horiba JY iHR-320) equipped with a cooled-charge coupled device (CCD) camera operated at $-100{ }^{\circ} \mathrm{C}$ (Andor DU920P) through the dichroic mirror, a pinhole and a long pass filter (Chroma HQ645LP). Accumulation time for all spectra were $10 \mathrm{~s}$. Measurements were carried out under ambient conditions and at room temperature.

\section{Results and discussion}

\subsection{Attachment of silver nanowire on W-tip}

Nanowires were first spin-casted onto a hydrophobized glass cover slide [Fig. 3(a)] (see Appendix A for details of the hydrophobization procedure). This aided the process of attachment by minimising the interaction between the PVP-coated nanowires and the substrate. Pre-etched W-tips (see Appendix B for the etching procedure) were then dipped into a droplet of quick-drying glue (Appendix C), which was achieved by using a micromanipulator whilst simultaneously recording a transmission image of the end of the tip. After observing a characteristic change in this image (i.e., decrease in transmittance), the tip was immediately withdrawn from the glue, allowing for a coating of between 50 and $100 \mu \mathrm{m}$ to be formed around the apex. Thereafter, the glue-coated tip was positioned above a prior selected nanowire [Figs. 3(a) and 3(b)], and was brought and held into contact for a period of $10 \mathrm{~s}$ [Fig. 3(d)]. The tip was finally retracted from the surface with the nanowire rigidly attached [Figs. 3(e) and 3(f)]. Figure 3(g) shows SEM image of a typical tip made using procedure. The nanowire was orientated in a parallel fashion relative to the W-tip, with the exposed region being around $4 \mu \mathrm{m}$ in length. A higher magnification image of a square drawn in Fig. 3(g) is shown in Fig. 3(h). Indeed, the exposed length could be precisely controlled by simply changing the position at which the apex of the W-tip comes into contact with the relevant nanowire.

It was not possible to achieve an ohmic electric contact between the two metals. Evidence supporting this is provided via SEM measurements, where it was found that some tips would break as a result of electrical charge build-up via the beam used for imaging. Different type of glues, such as a conductive epoxy that could alleviate such an issue, were found to be ineffective for 'picking-up' the nanowires. It is likely that the surface wetness between the glues and the tungsten surface are a key factor in this process.

The adhesion between the nanowire and the W-tip was significant. The probability of 
detachment after dipping the tip into solution was significantly reduced (less than 10\%) than when an alternative method (AC-DEP) was used (almost $50 \%$ tips detached) ${ }^{15,21)}$. This also has the advantage of increasing stability against vibration during TERS measurements.

\subsection{Attachment of nanoparticle onto silver nanowire}

Gold nanoparticle(s) could then be fixed in a site specific manner using AC-DEP. Dependent on the particular surfactant used, chemically-synthesised gold or silver nanoparticles exhibit either a positive or negative electrical charge $\mathrm{e}^{25)}$. Upon application of a DC (or AC) voltage between two electrodes, the particles can therefore be induced to move in a particular direction ${ }^{26)}$. The gold particles used here were coated in citric acid, meaning that they were negatively charged (Appendix D). A silver nanowire attached to a W-tip and a second pre-etched W-tip (both of which were electrically contacted) were immersed into a gold nanoparticle solution ( $50 \mu \mathrm{L}$ was dropped onto hydrophobized cover slip), for which the average diameter was approximately $100 \mathrm{~nm}$ [Figs. 4(a) and 4(b)]. The second tip was approached close to the nanowire (with a separation of between 1 and $2 \mu \mathrm{m}$ ) and an AC pulsed voltage was applied $\left(20 \mathrm{~V}_{\mathrm{p}-\mathrm{p}}, 80 \%\right.$ duty, $\left.1 \mathrm{KHz}\right)$ for $10 \mathrm{~s}$, leading to the application of a nanoparticle onto the nanowire at this point. Note that the probability to attach a nanoparticle was higher with AC pulsed voltage than with DC voltage. This might be due to larger heat generation with pulsed AC than with DC voltage, leading to higher attachment probability of the nanoparticles to the nanowire. Typical SEM images that could be obtained using this method can be seen in Figs. 4(c) and 4(d). The structure observed has a height and width of roughly 100 and $150 \mathrm{~nm}$, respectively, and is found at the position at which the counter electrode was placed. This therefore correlates well with the attachment of the gold nanoparticles.

Although this method is reliable -and allows for precise positioning-, the number of the nanoparticles found on the nanowire could not be fully controlled. This is most likely a result of both the difference in electrical conductivity found between the tips, as well as a variation in the separation between the two electrodes used in each case. Better conductivity and control of separation would allow for finer control of this method. Also it is noteworthy that this nanoparticle attachment process was not controllable with silver nanowire tips made with the method we have been used ${ }^{15,21)}$. During the AC-DEP process, nanowires often moved in solution - probably due to a longer exposed length and weaker adhesion between the silver nanowires and the tungsten surface (physisorbed) - resulting in lower reproducibility in nanoparticle attachment or indeed even in bubble formation due to heat 
generation induced by a short circuit.

\subsection{Remote image}

Rayleigh scattering was first measured, in order to evaluate whether the gold nanoparticle could act as a light coupling point. Laser light from a He-Ne laser (632.8 nm) was focused to the position of a gold nanoparticle [Fig. 5(a)], with the direction of polarization fixed perpendicular to the long axis of the nanowire. Figures 5(b) and 5(c) show a Rayleigh scattering image of the tip and intensity cross section at the dashed line in Fig. 5(b), respectively. As can be seen, emission was observed from the end of the nanowire, indicating photon-plasmon conversion has occurred by light irradiation at the nanoparticle position.

\subsection{TERS}

After verifying that emission from the nanowire end could be observed, the tip was then approached onto a benzenethiol-modified $\mathrm{Au}(111)$ surface under shear-force AFM feedback. This allowed for TERS spectra to be recorded and for direct and remote excitation to be compared. Again, a He-Ne laser was used as the excitation source, and was focused either to the end of the nanowire (direct excitation), or to the position of the gold nanoparticle (remote excitation), using both polarization parallel (p-polarization) and perpendicular (s-polarization) to the long axis of the nanowire. In the case of remote excitation, the detection spot (as determined by the pinhole position in our confocal setup) was fixed at the nanowire apex, with only the position of the excitation spot being moved.

Figures 6(a) and 6(b) show TERS spectra observed under direct excitation using p- (a) and s- (b) polarization with a laser power of $50 \mathrm{~kW} / \mathrm{cm}^{2}$. The Raman peaks at 700, 999, 1023, 1072 , and $1572 \mathrm{~cm}^{-1}$ are resolved in the spectra, which can be assigned to the vibrational modes of C-H out-of-plane deformation, a mixture of S-H bending and in-plain ring deformation, in-plane ring deformation, a mixture of C-S stretching and in-plane ring deformation, and $\mathrm{C}-\mathrm{C}$ stretching of benzenethiol, respectively. It was found that both the Raman and background intensities were higher with p-polarization than with s-polarization, resulting from the excitation of gap-mode plasmons between the apex of the silver nanowire and the $\mathrm{Au}(111)$ substrate ${ }^{14,27)}$. Conversely, with remote excitation this polarization dependence was reversed. Figures 6(c) and 6(d) show TERS spectra observed under remote excitation with p- and s-polarization, respectively, using the same laser power $\left(50 \mathrm{~kW} / \mathrm{cm}^{2}\right)$. The spectra in Figs. 6(c) and 6(d) are multiplied by 10 times for easier comparison. Note that, 
with remote excitation, when the laser power is above $100 \mathrm{~kW} / \mathrm{cm}^{2}$, the remote-TERS signal tends to fluctuate and indeed sometimes the nanowire was cut at the focused spot. This could be due to a temperature rise at the nanoparticle/nanowire junction upon laser irradiation. Here, both the Raman and background intensities were higher with s-polarization than with p-polarization. This is in line with expectation, given the fact that light polarized perpendicular to the long axis of a nanowire can more efficiently excite propagating surface plasmons when a laser is irradiated at a coupling point ${ }^{28)}$. This stronger coupling therefore leads to a stronger excitation of propagating surface plasmons travelling along the nanowire, which can thus explain the above observation.

Generally, the resolution that can be expected in a TERS experiment is directly related to the enhancement factor, EF, given by ${ }^{29,30)}$

$$
E F \approx\left(\frac{I_{\text {tip-in }}}{I_{\text {tip-out }}}-1\right) \frac{A_{F F}}{A_{N F}},
$$

where $I_{\text {tip-in }}$ and $I_{\text {tip-out }}$ are Raman peak intensities measured with the tip in contact and retracted, $A_{F F}$ is the area of the far-field laser spot, and $A_{N F}$ is the effective area of the TERS spot (which can be estimated from the diameter of the tip apex). From this, it can be seen that any Raman scattering or photoluminescence emanating from the region under the laser spot, but not from the region under the tip apex, will contribute to the TERS spectrum as $I_{\text {tip-out }}$. As a result of this, any scatterer or emitter on the sample will typically contribute as a broad background emission and so decrease the obtainable sensitivity in a TERS image or spectrum.

However, in the case of remote excitation, this background contribution from the sample should be lower as the excitation spot is no longer focused onto the surface. Indeed, lower background intensities have been observed with remote excitation relative to direct excitation, demonstrated by both our group and other researchers ${ }^{19-23)}$. A notable example of this is given by Berweger, who created a grating structure as a light coupling point on a chemically etched gold $\operatorname{tip}^{22,23)}$. As here, this resulted in a vertical separation of the excitation and detection spots, and led to a lower background intensity than when the laser was focused to the tip apex.

To assess this more quantifiably, background intensities of TERS spectra were compared. Figure 7 shows normalised spectra (at Raman intensity of C-C stretching peak, $1572 \mathrm{~cm}^{-1}$ ) of the direct excitation with p-polarization, remote excitation with s-polarization, and without the tip. Note that the peak height of the Raman scattering at $1572 \mathrm{~cm}^{-1}$ was estimated in each case by fitting a Lorentzian function. As can clearly be seen, the TERS spectrum with remote 
excitation exhibits a lower background than in the other two cases. The relative background intensity is highest in the spectra where no tip is present (roughly 3 and 4 times higher than direct and remote excitation, respectively), possibly as a result of inelastic scattering/photoluminescence from surface contamination or cluster-like structures that might exist on the substrate. From these results, we conclude that the fabricated tips show activity when remote excitation is used, increasing the sensitivity in TERS.

\section{Conclusions}

We have fabricated a new type of tip which can be suitable for remote excitation-TERS measurements. The tip is made by fixing a single silver nanowire onto a W-tip using a micromanipulator equipped onto an optical microscope, after which a gold nanoparticle is site-specifically attached onto the body of the silver nanowire using AC-DEP. Compared to an older generation tip $^{15,21)}$, the exposed length of the silver nanowire and the position of the light coupling point (nanoparticle-attached point) are fully controllable. TERS spectra measured when laser light is focused onto a nanoparticle position showed lower background intensity than when the light is focused onto the nanowire apex, proving the remote activity. Since the tip is based on chemically-synthesized silver nanowires, a high reproducibility in TERS measurements is maintained. In addition, since the fabrication process does not require any cost-consuming equipment, e.g., focused ion beam milling, remote excitation can be realized in a cost-effective manner, thereby increasing the applicability of such tips. Furthermore, although we have demonstrated the remote activity using TERS microscopy, we believe that this type of tip can be also useful for finer nanowire-based three-dimensional (3D) SERS microscopy, which we have recently demonstrated on a living cell ${ }^{21)}$.

In this paper, we have shown state of the art remote-TERS activity by simply comparing spectra obtained using direct and remote configurations. However, further fundamental studies, such as a conclusive demonstration of the improvement in spatial resolution, or a determination of the dipole orientation of the surface plasmons at the nanowire end, would be of importance in order to widen the applicability of the technique. In addition, we found that the TERS signal for remote excitation was usually 10-15 times weaker than that of direct excitation. In order to therefore increase the TERS sensitivity still further, the use of structures that give a higher light coupling efficiency and prolong the propagation length of the surface plasmons would also merit investigation.

\section{Acknowledgments}


This work was supported by the Fonds voor Wetenschappelijk Onderzoek FWO (G.0B55.14, G081916N, G.0B94.13, and G.0259.12) and the K.U. Leuven Research Fund (BOF-OT and BOF-C14). The financial support of the Belgian Federal Science Policy Office (IAP-VI/27), Morino fundation and the Japan Science and Technology Agency PRESTO program are gratefully acknowledged. The research leading to these results has also received funding from the European Research Council under the European Union's Seventh Framework Programme (FP7/2007-2013)/ERC Grant Agreement PLASMHACAT (No. 280064) to HU and Grant Agreement NANOGRAPH@LSI (No. 340324) to SDF.

\section{Appendix}

\section{Appendix A: Hydrophobization of cover slip}

$30 \mathrm{uL}$ of Hexyltrimethoxysilane was dropped into a small glass beaker. A prior cleaned cover slip was placed on top of a beaker, which was then heated to $80{ }^{\circ} \mathrm{C}$ for $15 \mathrm{~min}$. After this, the cover slip was washed with MilliQ and then dried with $\mathrm{N}_{2}$ gas. This process was repeated 2 times.

\section{Appendix B: Etching of W-tip}

Approximately 1-2 mm of a Tungsten wire ( $\phi 0.05 \mathrm{~mm}$, ADVENT) was dipped into a $2 \mathrm{M}$ $\mathrm{NaOH}$ solution. A 2.5 V AC-voltage was then applied between the tungsten wire and a $\mathrm{Pt}$ counter electrode, before being stopped immediately on etching to prevent any over-etching to the wire. Finally, the etched-tip was washed with acetone and MilliQ.

\section{Appendix C: Glue}

Super glue (Loctite SUPER GLUE-3) was used. The main ingredient of this glue is cyanoacrylate. This glue reacts with water when it is polymerized. The use of similar types of glue (Konishi Aron Alpha) also worked for pick-up.

\section{Appendix D: Gold nanoparticle}

Gold nanoparticles having a diameter of $100 \mathrm{~nm}$ were purchased from Nanopartz (AK11-100-25mL). 


\section{References}

1. M. Inaba, Y. Kawatate, A. Funabiki, S. Jeong, T. Abe and Z. Ogumi, Electrochim. Acta 45, 99 (1999)

2. Y. Takahashi, A. Kumatani, H. Munakata, H. Inomata, K. Ito, K. Ino, H. Shiku, P. R. Unwin, Y. E. Korchev, K. Kanamura and T. Matsue, Nat. Commun. 5, 5450 (2014)

3. N. Balke, S. Jesse, A. N. Morozovska, E. Eliseev, D. W. Chung, Y. Kim, L. Adamczyk, R. E. García, N. Dudney and S. V. Kalinin, Nat. Nanotechnol. 5749 (2010)

4. M. Shih, B. Huang, C. Lin, S. Li, H. Chen, Y. Chiu and C. Chen, Nano Lett. 13, 2387 (2013)

5. O. Stetsovych, M. Todorović, T. K. Shimizu, C. Moreno, J. W. Ryan, C. P. León, K. Sagisawa, E. Palomares, V. Matolin, D. Fujita, R. Perez and O. Custance, Nat. Commun. 6, 7265 (2015)

6. Y. Wang, H. Sun, S. Tan, H. Feng, Z. Cheng, J. Zhao, A. Zhao, B. Wang, Y. Luo, J. Yang and J. G. Hou, Nat. Commun. 4, 2214 (2013)

7. C. Bai, Scanning Tunneling Microscopy and Its Application (Springer, New York, 2000) 2nd ed., p. 2.

8. J. Wessel, J. Opt. Soc. Am. B 2, 1538 (1985)

9. R. M. Stöckle, Y. D. Suh, V. Deckert and R. Zenobi, Chem. Phys. Lett. 318, 131 (2000)

10. N. Hayazawa, Y. Inouye, Z. Sekkat and S. Kawata, Opt. Commun. 183, 333 (2000)

11. M. S. Anderson, Appl. Phys. Lett. 76, 3130 (2000)

12. B. Pettinger, G. Picardi, R. Shcuster and G. Ertl, Electrochemistry 68, 942 (2000)

13. L. G. Cancado, A. Hartschuh and L. Novotny, J. Raman Spectrosc. 40, 1420 (2009)

14. J. Steidtner and B. Pettinger, Phys. Rev. Lett. 100, 236101 (2008)

15. Y. Fujita, R. Chiba, G. Lu, N. N. Horimoto, S. Kajimoto, H. Fukumura and H. Uji-i, Chem. Commun. 50, 9839 (2014)

16. T. Ichimura, N. Hayazawa, M. Hashimoto, Y. Inouye and S. Kawata, Phys. Rev. Lett. 92, 220801 (2004)

17. R. Zhang, Y. Zhang, Z. C. Dong, S. Jiang, C. Zhang, L. G. Chen, L. Zhang, Y. Liao, J. Aizpurua, Y. Luo, J. L. Yang and J. G. Hou, Nature 498, 82 (2013)

18. C. Zhang, B. Chen and Z. Li, J. Phys. Chem. C 119, 11858 (2015)

19. J. A. Hutchison, S. P. Centeno, H. Odaka, H. Fukumura, J. Hofkens and H. Uji-i, Nano Lett. 9, 995 (2009)

20. J. A. Hutchison and H. Uji-i, in Review in Plasmonics, ed. C. D. Geddes (Springer International Publishing, Cham, 2015) p. 417.

21. G. Lu, H. De Keersmaecker, L. Su, B. Kenens, S. Rocha, E. Fron, C. Chen, P. Van Dorpe, H. Mizuno, J. Hofkens, J. A. Hutchison, H. Uji-i, Adv. Mater. 26, 5124 (2014)

22. S. Berweger, J. M. Atkin, R. L. Olmon and M. B. Raschke, J. Phys. Chem. Lett. 1, 3427 (2010)

23. S. Berweger, J. M. Atkin, X. G. Xu, R. L. Olmon and M. B. Raschke, Nano Lett. 11, 4309 (2011) 
24. M. Rycenga, C. M. Cobley, J. Zeng, W. Li, C. H. Moran, Q. Zhang, D. Qin, Y. Xia, Chem. Rev. 111, 3669 (2011)

25. C. Kaulen, M. Homberger, S. Bourone, N. Babajani, S. Karthäuser, A. Besmehn and U. Simon, Langmuir 30, 574 (2014)

26. B. C. Gierhart, D. G. Howitt, S. J. Chen, R. L. Smith and S. D. Collins, Langmuir 23, $12450(2007)$

27. Y. Fujita, N. N. Horimoto, S. Kajimoto and H. Fukumura, Chem. Phys. Lett. 584, 110 (2013)

28. H. Wei, F. Hao, Y. Huang, W. Wang, P. Nordlander and H. Xu, Nano Lett. 8, 2497 (2008)

29. N. Kumar, A. Rae and D. Roy, Appl. Phys. Lett. 104, 123106 (2014)

30. N. Kumar, S. Mignuzzi, W. Su and D. Roy, EPJ Tech. Instrum. 2, 1 (2015) 


\section{Figure Captions}

Fig. 1. Schematic diagram of conventional excitation TERS configuration (direct excitation TERS) (a), and remote excitation TERS using gold nanoparticle-attached silver nanowire tip (b).

Fig. 2. Schematic of the TERS setup.

Fig. 3. (a) Schematic of the deposition process of silver nanowires onto a hydrophobized-coverslip. (b)-(f) Transmission images during attachment of a silver nanowire to pre-etched W-tip; the W-tip is positioned several micrometers above a target nanowire (b, c), and is then brought into contact with the nanowire (d), before finally being retracted from the surface $(e, f)$. ( $g, h)$ Typical SEM images of a fabricated nanowire tip. Right figure (h) shows higher magnification image of the square drawn in $(\mathrm{g})$.

Fig. 4. (a) Schematic of the gold nanoparticle(s) attachment process to a silver nanowire tip via AC-DEP. (b) Transmission images taken during the AC-DEP process. (c, d) SEM images after AC-DEP. Right figure (d) shows a higher magnification image at the square drawn in (c).

Fig. 5. (a) Schematic of the excitation configuration when a Rayleigh scattering image was obtained. (b) Rayleigh scattering image of a fabricated tip. Laser light from He-Ne laser $(632.8 \mathrm{~nm})$ is focused at the gold nanoparticle-attached point on a silver nanowire under polarization perpendicular to the long axis of the nanowire. (c) Cross section at white dashed line drawn in (b).

Fig. 6. Raman spectrum from benzenethiol-modified $\mathrm{Au}(111)$ substrate in the presence (a)-(d), and the absence of the tip (e). (a, b) TERS spectra measured when He-Ne laser light $\left(632.8 \mathrm{~nm}, 50 \mathrm{~kW} / \mathrm{cm}^{-1}\right)$ is focused at the end of a silver nanowire with $\mathrm{p}$ - (a) and s-polarization (b) (direct excitation). (c, d) TERS spectra measured when the laser light is focused at the gold nanoparticle with p- (c) and s-polarization (d) (remote excitation). The tip is approached to the substrate under shear-force AFM feedback. Spectra shown in (c)-(e) 
are multiplied 10 times for easier comparison. Raman peaks that are assigned to Raman scattering of benzenethiol are indicated by an arrow in (a).

Fig. 7. Normalized spectra (at $1572 \mathrm{~cm}^{-1}$ (C-C stretching mode of benzene ring), indicated by green background) measured under direct excitation with p-polarization (red), under remote excitation with s-polarization (blue), and without tip (black). The Normalization process allows for direct comparison of background intensity between spectra. 
Figure 1 (Color Online)

\section{(a) Direct excitation}

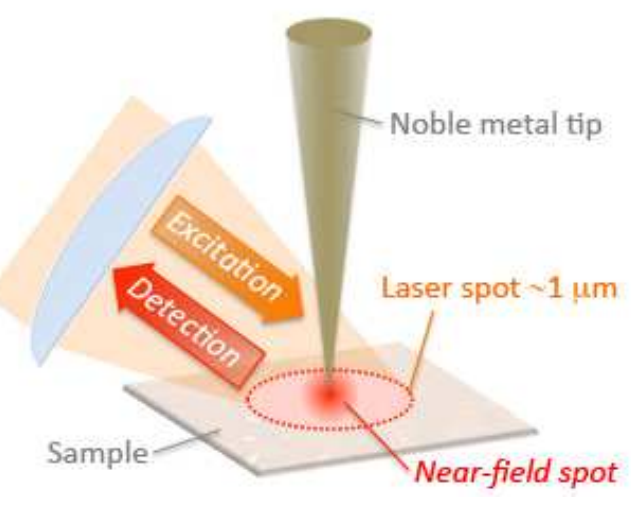

(b) Remote excitation

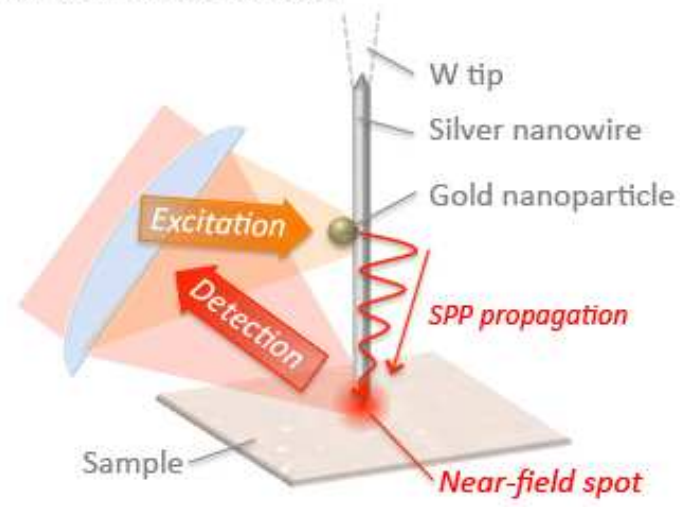


Figure 2 (Color Online)

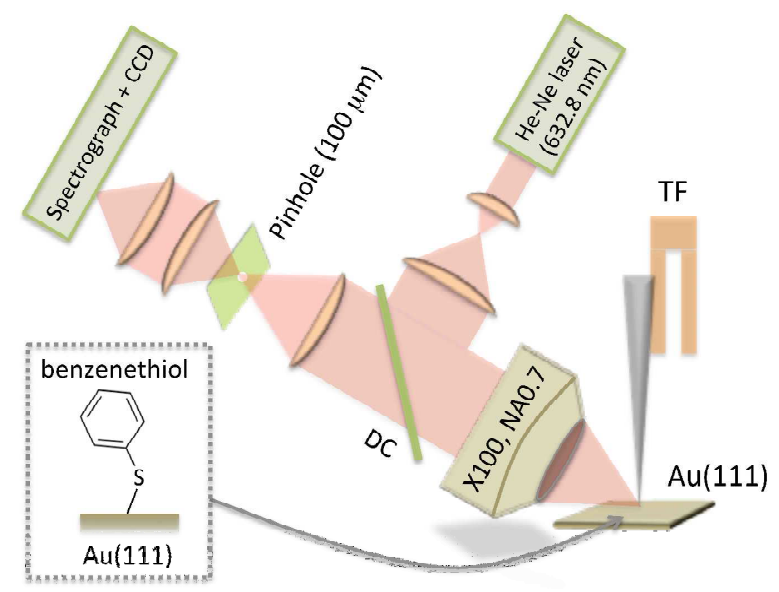


Figure 3 (Color Online)
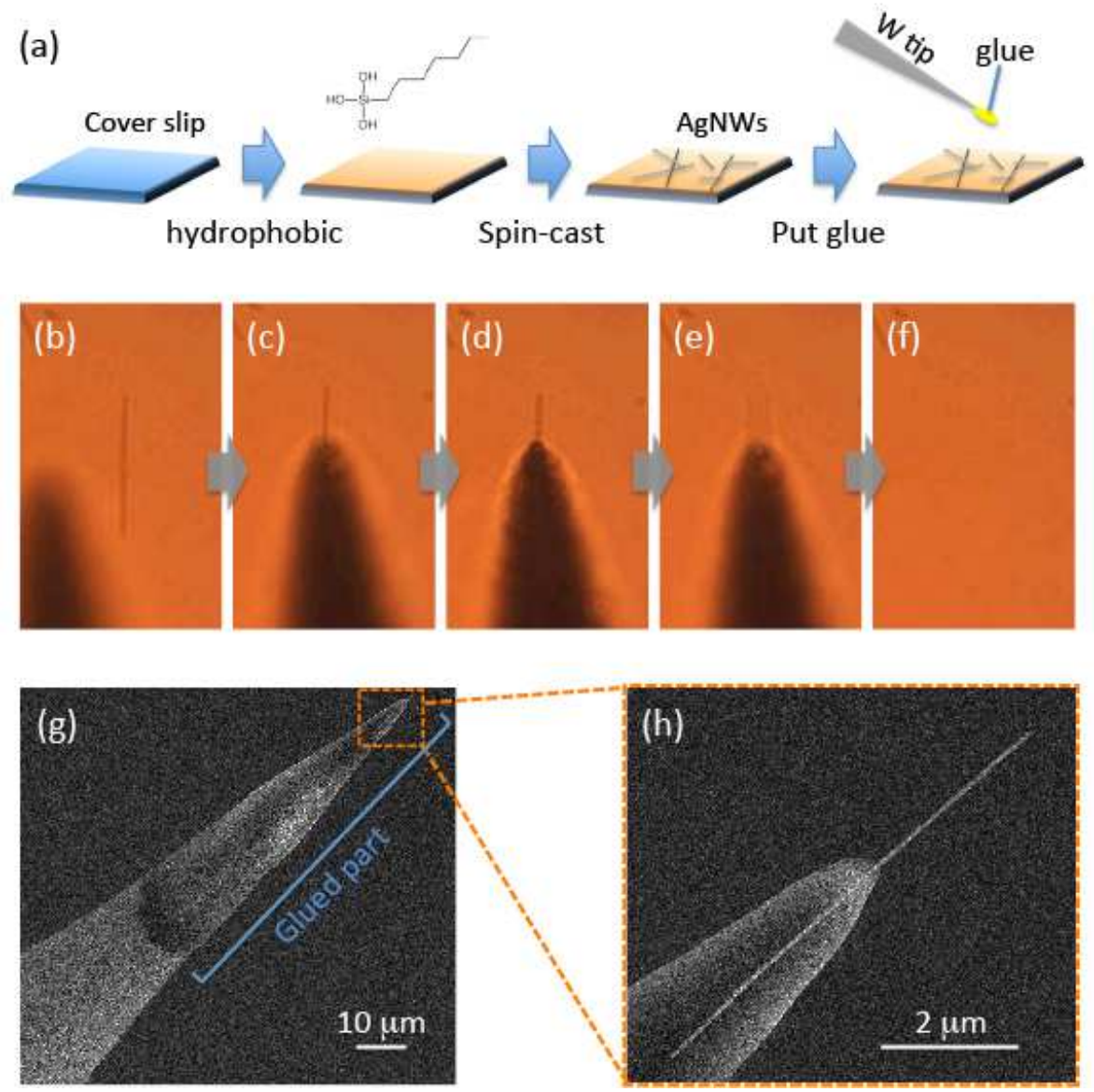
Figure 4 (Color Online)

(a)

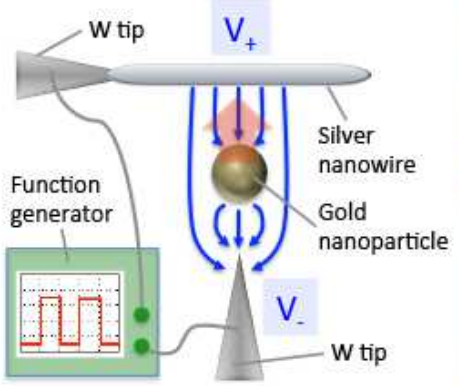

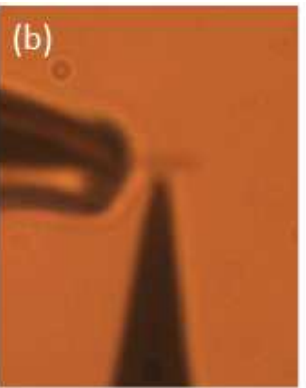

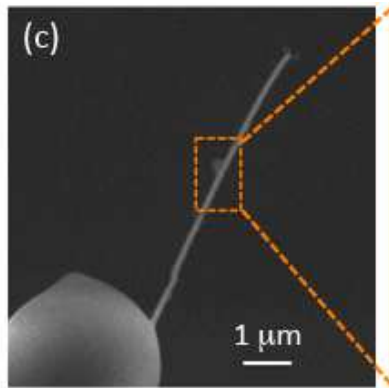


Figure 5 (Color Online)

(a)

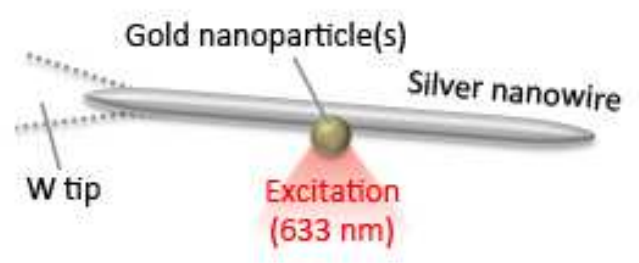

(b) Remote image

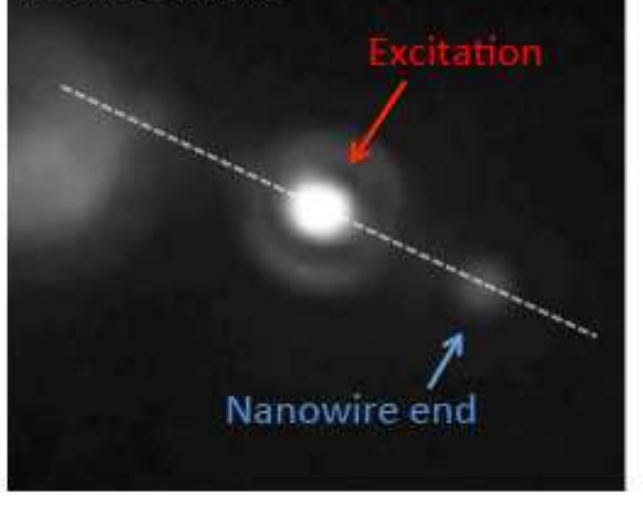

(c) 
Figure 6 (Color Online)

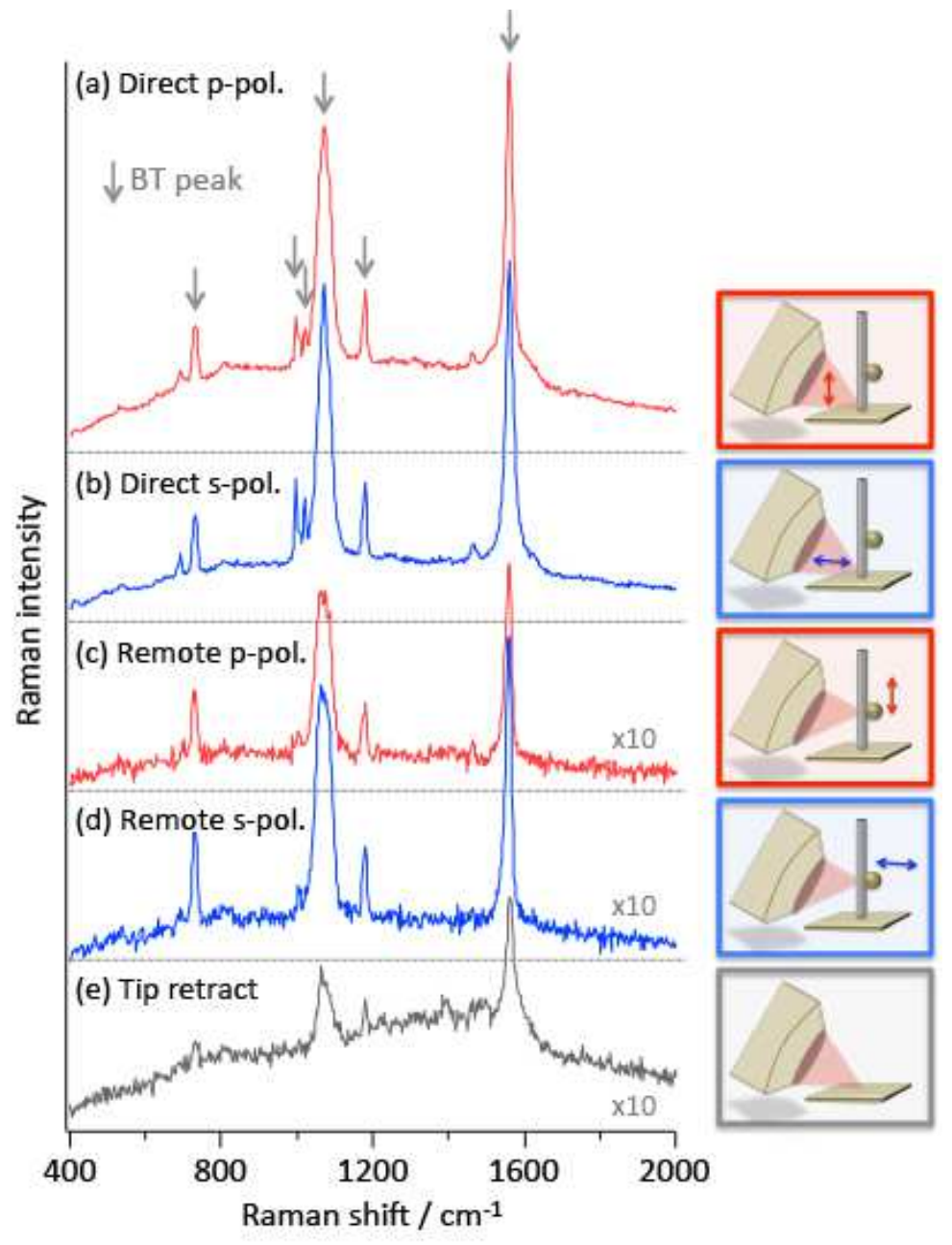


Figure 7 (Color Online)

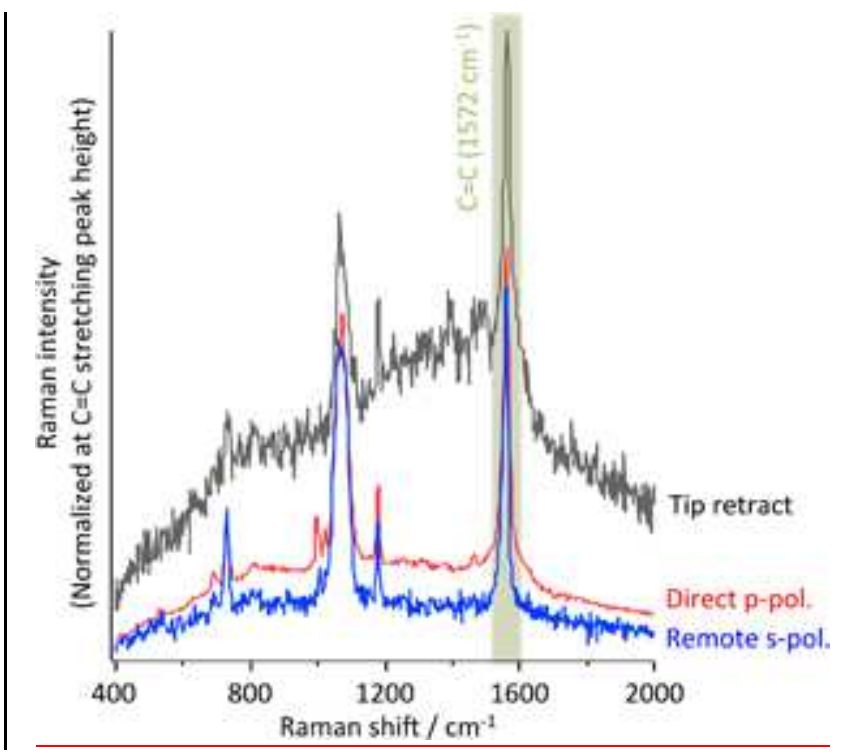

